\title{
Application of the Hough Transform for Weld Inspection Underwater
}

\author{
Alistair R. Greig \\ Department of Mechanical Engineering, University College London, \\ University of London, Torrington Place, LONDON, WC1E 7JE, UK.
}

\begin{abstract}
The inspection of the nodal welds of off-shore steel structures is a necessary but hazardous task at present performed at regular intervals by divers. Automation of this task is an on-going research effort. One requirement for automation is the accurate location of the weld roots and it is this which is considered here. A brief description of the system is provided as well as a summary of the problems of imaging underwater. This is followed by a description of how the relevant segments of the degraded laser stripe can be identified using the Hough transform. The Rainflow method (originally proposed by Tatsuo Endo for analysis of stress histories) is then used to rapidly identify the desired features on the parameter surface and hence locate the weld roots.
\end{abstract}

\section{Introduction}

The welds joining the main structural members of steel jacket, off shore platforms can suffer from fatigue cracks due to wave loading on the platform. Inspection is currently done by divers but there is a requirement to automate this [1]. To successfully examine the large, multi-pass welds found at the junction of the tubular structural members it is necessary to locate the weld roots, (the edges of the weld where cracks are most likely to occur). One proposed method for achieving this is by using a manipulator deployed machine vision system.

\section{Imaging System}

Automatic welding machines which track the weld run are now available from various manufacturers. These use a variety of methods for tracking the weld run, but all rely either on feed back from the weld arc, or the strong visual cues available from the prepared edges of the metal. Neither of these information sources are available when attempting to track an existing weld. The weld and surrounding metal are bland and devoid of useful features, especially if any corrosion has taken place. Marine growth may also be present when inspecting underwater, though most of this will have been removed by grit blasting.

Since no features are readily available a structured light source is introduced. A laser line is projected onto the weld and viewed at an angle by a CCD camera. This produces a profile of the weld and its position can easily be identified by triangulation. In air the laser line projected on to the metal surface is sharp, continuous, clear and easily detected by simple thresholding, see Fig. 1. The weld roots are easily identifiable as the ends of the upper and lower line segments.

The imaging system is mounted on the end of a manipulator which is used to move it along the weld. An initial approximation of the weld path is constructed by assuming that the structural members are cylinders and that the weld occurs at their intersection. As the imaging system profiles the weld and identifies the roots, the results are feed back to the manipulator path planning routine to improve its performance. Unlike land based robotic applications, speed of operation is not a priority for underwater operation. 


\section{Imaging Underwater}

An underwater image will be degraded by the total attenuation due to the transmission of light through the sea water, and also in this case by the debris after cleaning. There are a number of mechanisms which contribute to the degradation of an image captured underwater, fuller descriptions are available elsewhere $[2,3,4]$, but in summary;

- Molecular scattering. Pure water will scatter light in all directions, though the distribution is not uniform, the ions in sea water increase scattering (typically $30 \%$ ).

- Particle scattering. Particles are small $(1-100 \mu \mathrm{m}$ diameter) pieces of organic or inorganic material held in suspension in the water, these will also scatter light but scattering is predominantly forward. Particle concentration and size distribution depends on geographical location, weather conditions and depth.

- Absorption. Light is absorbed by the water itself and also the organic matter dissolved in it. This is very wave length dependant and minimum absorption occurs in a narrow band $(460-560 \mathrm{~nm})$ depending upon the concentration of organic matter.

By reducing the operating range to less than $1 \mathrm{~m}$ and using a green laser the above effects can be reduced. Even so the laser line will not be as sharply defined as in air. Also rigs produce their own micro-marine environments and act like artificial reefs, consequently the biological and suspended matter will be much higher than might be expected than for that particular sea area. Before inspecting the weld area it is necessary to grit blast the soft organic and hard calcareous growth off from the structure, this produces an additional problem of large (diameter $>100 \mu \mathrm{m}$ ), inorganic particulate matter floating in the viewing volume. These particles are too large to cause significant scattering but depending upon their orientation and position they can either obscure part of the image or create bright spots by reflecting the laser.

The combination of the above effects produces an image that is blurred and the occlusions will cause the stripe to be discontinuous. After thresholding the line will be surrounded by a number of bright spots due to reflections from the debris. Once the laser stripe is identified within the image it is relatively straight forward to track as it will not vary significantly form one frame to the next as the manipulator's motions are slow. There are two exceptions to this. Large portions of the stripe will occasionally be obscured when a large piece of debris floats (swims) through the viewing volume. As the manipulator moves around the weld it will occasionally have to reconfigure itself to avoid a singularity transit or a joint limitation. This will necessitate moving the laser stripe away from the weld. A reduced version of the Hough transform is used to rapidly identify the line segments for the initial stripe detection or when ever its location is lost.

\section{Application of Hough Transform}

The Hough transform is a method for identifying sets of co-linear points on an image. Description of the method is available in many standard image processing texts $[5,6]$. It is recognised that the method has a number of limitations;

- It is slow to implement

- To achieve good resolution a large matrix is required to represent parameter space.

- The extraction of features from the parameter space can be very time consuming.

For this application a priori knowledge is used to speed implementation of the transform. Location of the local maxima in the parameter plane is simplified by reduction of the problem from three to two dimensions and by subsequent use of the rainflow method to extract features.

Hough's original implementation of his transform [7] used the gradient/intercept parametrisation of a straight line $(y=m x+c)$, subsequently Duda [8] suggested the normal parametrisation $(x \operatorname{Cos} \theta+y \operatorname{Sin} \theta=r)$. The latter form is now generally used for Hough transforms. The normal form has the advantage that it provides a bounded 
parameter space, but the disadvantage that it is slower to plot, straight lines are far quicker to compute than sinusoids. By controlling the path of the manipulator around the weld and the pose of the camera relative to the laser it was possible to restrict the orientation of the laser stripe. The gradients of all its significant line segments were within a limited, finite range, $-1.28 \leq m \leq 1.28$. Constraining the gradient values removes the limitation on using the gradient/intercept parametrisation as a closed solution is now possible. This permits faster generation of the transform.

The Hough transform is generated on the image plane of the image processing board rather than using a large $(0.74 \mathrm{Mb})$ matrix within the host computer, each pixel acts as a collection bin. $f(x, y)=h(c, m)$. The value (gray scale) of each pixel is set to zero and the ink colour to one. The parameter plane is then generated by drawing lines on the plane with the logical operator set to AND. The limiting factor on speed is the time taken to interrogate the original image in the frame store, not the time taken to construct the transform. This approach has the advantage that a very large number of bins $(512 \times 720)$ can be used at virtually no extra cost in computing effort.

Two points are sought on the parameter plane, corresponding to the upper and lower line segments. Both points will be local maxima and will have different values of $m$ as the line segments will always have different gradients. As the system is operating in an unstructured environment atypical conditions must be considered. It is possible for the local maximum associated with the weld line segment to be of similar magnitude to that of one of the other line segments, therefore it is also necessary to identify the third most significant maximum on the parameter plane. This will be a rare occurrence as the weld line segment is usually shorter and less regular than the other two line segments. Even when this condition does occur, in the majority of cases the ambiguity can be resolved by considering the indicated positions of the lines on the image plane. If the result is still ambiguous two steps of remedial action are taken before halting the programme. On the assumption that the error was caused by matter within the viewing volume a fresh image is captured and the procedure repeated. If this fails the manipulator repositions the laser stripe in what is hoped to be a better position over the weld. The manipulator is moved so as to reduce the length of the one easily identifiable line segment with the intention to increase the length of the other.

The relative strength of the weld line segment can be reduced by viewing weld from further away, this increases the length of the upper and lower line segments. Increasing the viewing range has three disadvantages; loss of resolution, increased attenuation and scattering, increased line curvature. The upper and lower line segments are not straight lines but arcs of an ellipse, the aspect ratio of the ellipse is such that over short arc lengths the curvature is nominal. The manipulator is programmed to orientate the laser to minimize this effect. The Hough transform provides a best line fit to the arc.

The geometry of the cylinders and the manipulator's path ensure the upper and lower line segments always have different gradients. Hence the local maxima search on the parameter plane can be reduced from considering all the 368,640 cells of $h(c, m)$ to just the 512 elements of $h\left(c_{\text {Max }}\right)$. For each gradient value $m$ the maximum value of $c$ is found. The resulting function is not smooth due to noise on the original image.

The problem is to extract the position of the three distinct maxima. It is not sufficient to find the three highest peeks of the function $h\left(c_{M a x}\right)$ as these are usually clustered around a narrow band and relate to a single line on the image plane. Consider Fig. 2; A, B and C are the peaks of interest, but 1,2 and 3 are the three largest maxima. A filtering method is required that identifies large amplitude cycles and does not corrupt the original data pertaining to them as is the case with smoothing functions. This was achieved using the rainflow method. This method has the advantage over many filtering and thresholding techniques in that it does not require the values of any coefficients or limit values to be preset. The correct and reliable evaluation of the coefficients and limit values for filters is often a limitation on their effectiveness, especially when operating in environments with poorly controlled conditions. 
The rainflow method was developed for the study of stress histories of randomly loaded structures [9]. The method successively filters out small stress reversals to leave only those of large amplitude. It is these that cause the majority of damage to structures and are so important for fracture mechanics and fatigue analysis. The rainflow method preserves amplitude information and operates independently of frequency.

The rainflow method is best explained by means of a physical analogy. The variant presented here is sometimes referred to as the reservoir method. It has the advantage that it identifies the largest rather than the smallest cycles first. Figure 3a shows the simplified function $y=f(x)$. The two local maxima with the greatest magnitude are $\mathrm{A}$ and $\mathrm{B}$ while the peaks of the two main cycles are at $\mathrm{A}$ and $\mathrm{C}$. The function is considered as a continuous loop and rearranged about $x[\mathrm{~min}]$, Fig. $3 \mathrm{~b}$, so as to include any maxima at the limits of $x$, for example D. Consider a reservoir of buoyant gas trapped beneath the function, find the absolute maximum and let the gas escape at that point, the result is shown in Fig. 3c. The next most significant cycle can be identified by considering the depth of gas trapped beneath it, as can be seen from Fig. 3d. The process is repeated as required, note that B corresponds to only the fourth most significant cycle.

The method is rapid as it requires little computation beyond simple hill climbing and sort routines. It becomes progressively faster as the most significant cycles are progressively identified and removed. The example here is for a two-dimensional system, the principle can be extended to three-dimensions for consideration of the full parameter plane. Work has been done on multi-axial loading of structures [10] which is relevant, the main problem is analysis of the values at the edges of the parameter surface.

\section{Results}

Tests were conducted on a $1 / 4$ scale tubular Y-joint, the camera-laser system being deployed from a PUMA 560 manipulator. The tests were conducted in air as neither an underwater camera nor an underwater manipulator were available. Access to underwater test facilities is a serious limitation to research in off-shore engineering. To simulate underwater conditions the line optics were defocused and noise was deliberately superimposed onto the image. The image is randomly seeded with two populations of blobs. One population, which covers $5 \%$ of the total image is bright (gray scale 255) while the other which covers $10 \%$ of the image is dark (gray scale 0 ).

The system was able to track all parts of the weld around the Y-joint. The example shown in Fig. 4 is a worst case situation taken at the toe of the weld, at this position the angle subtended between the upper and lower line segments is a minimum. The weld is approximately $30 \mathrm{~mm}$ wide at this point. Note the added complications of reflections from the metal surface (top centre), and the ghost stripe to the right of the main stripe due to internal reflections in the optics.

Figure 5 shows detail of the weld area with the results of the inverse transform and root location superimposed. In this particular case the near vertical, dark grey, line which corresponds to the upper line segment was the third most significant on the parameter surface. The most significant line being the light grey line which includes large proportions of the weld segment and the upper line segment. Despite this the upper weld root (Root 1) has been correctly located as has the lower weld root. The broken, light gray line was the second most significant feature on the parameter plane and corresponds to the lower line segment. Note that the lower root location is not assumed to be at the intersection of the lines, though this is often the case. 


\section{Conclusions}

A system has been constructed and tested that can detect and track the roots of a weld. It is able to operate in an optically harsh environment with serious image disturbances. Suitable design of the image system and deployment strategy permit simplification (and hence acceleration) of image processing. A simplified version of the Hough transform is successfully implemented, using the rainflow (or reservoir) method to assist in feature extraction from the parameter surface. At present the accuracy of the system is compromised by the accuracy of the deploying manipulator.

\section{References}

1. Bennett, A. and Chadwick, F.J. (1988). Crack detection on subsea structures - a cost effective approach. IEE Economics of NDE symposium, London 1988.

2. Duntley, S.Q. (1963). Light in the sea. J. Opt. Soc. Am. Vol. 53, pp. 214 - 233.

3. Morel, A. (1974). Optical properties of pure water and pure sea water. In: Optical aspects of Oceanography (Jerlov, N. and Nielsen, E.S. eds.), pp. 1 - 24. London: Academic Press. ISBN 0-12-384950-0.

4. Jerlov, N.G. (1976) Marine Optics. Elsevier Oceanography Series, 14. Amsterdam: Elsevier Scientific Publishing Company. ISBN 0-444-41490-8.

5. Gonzalez, R.C. and Wintz, P. (1987). Digital Image Processing $2^{\text {nd }}$ Ed. pp $130-136$. Reading MA: Addison-Wesley, ISBN 0-201-11026-1.

6. Leavers, V.F. (1992). Shape detection in Computer Vision Using the Hough Transform, London: Springer-Verlag ISBN: 3-540-19723-0.

7. Hough, P.V.C. (1962). Methods and means for recognizing complex patterns. U.S. Patent $3,069,654$.

8. Duda, R.O. and Hart, P.E. (1972). Use of Hough transforms to detect lines and curves in pictures. Comm. Association for Computing Machinery 15, No. 1, pp. 11 - 15.

9. Nelson, D.V. (1991). Rainflow and ordered overall range cycle counting, in The rainflow method in fatigue, the Tatsuo Endo memorial volume, Ed. Murakami, Y. pp. 41 - 50. Oxford: Butterworth Heinemann, ISBN 0750605049.

10. Beste, A., Dreßler, K., Kötzle, H., Krüger, W. Maier, B. and Petersen, J. (1991). Multiaxial rainflow, a consequent continuation of professor Tatsuo Endo's work, in The rainflow method in fatigue, the Tatsuo Endo memorial volume, Ed. Murakami, Y.pp. 31 - 40. Oxford: Butterworth Heinemann, ISBN 0750605049.

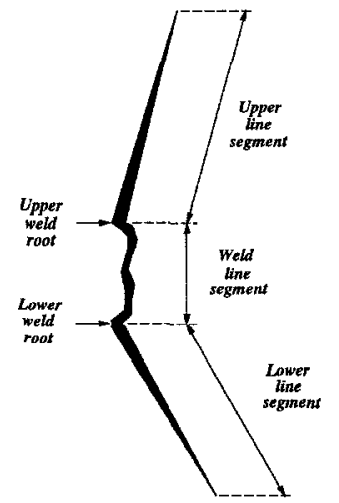

Fig. 1. Typical weld profile.

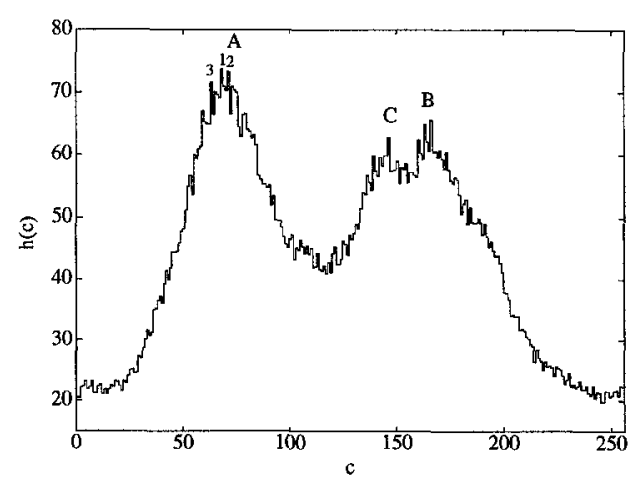

Fig. 2. Reduced parameter data set. 


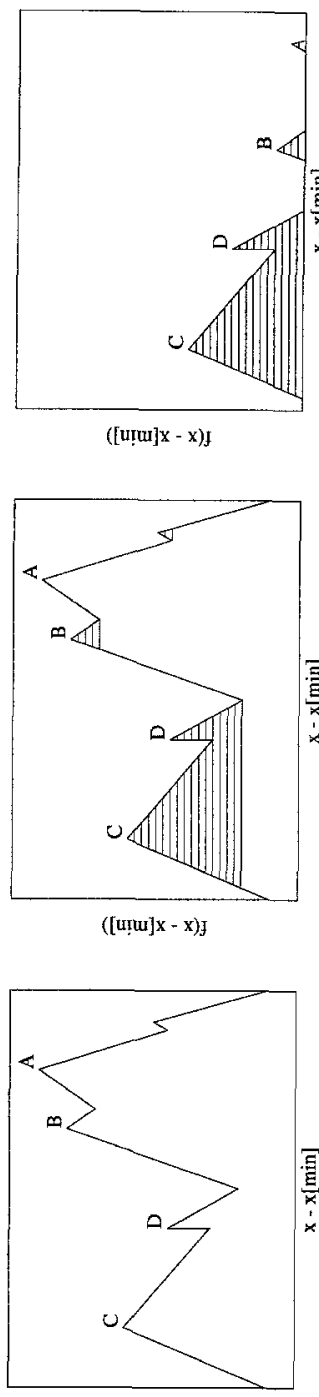

([u!̣]x - x)]

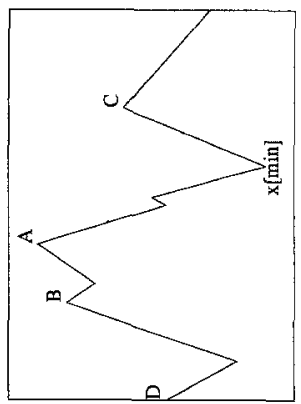

(x)]

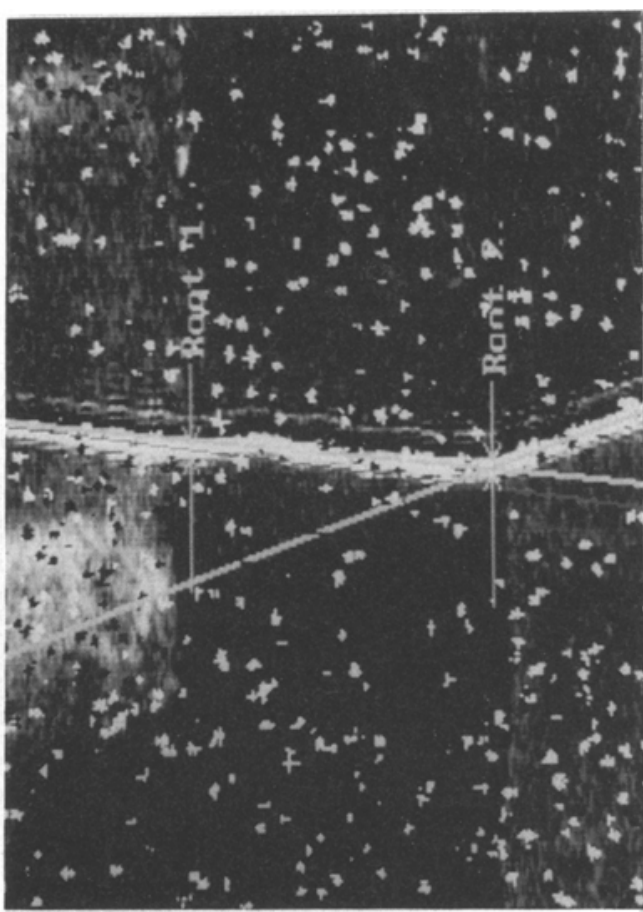

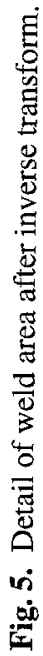

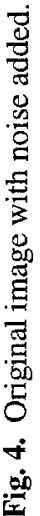

